\title{
Comprehensive Quantitative Elemental Microanalysis with Electron-Excited Energy Dispersive X-ray Spectrometry (EDS): 50 Years Young and Getting Better Every Day!
}

Dale E. Newbury ${ }^{1}$ and Nicholas W. M. Ritchie ${ }^{1}$

1. National Institute of Standards and Technology, Gaithersburg, MD.

50 years of intensive development of electron-excited X-ray microanalysis with energy dispersive X-ray spectrometry (EDS) has created an analytical tool that is now capable of comprehensive quantitative elemental characterization of microstructures [1,2]. By "comprehensive" we note that:

1. With the exceptions of $\mathrm{H}$ and $\mathrm{He}$ which do not produce $\mathrm{X}$-rays, all other elements of the Periodic Table can now be measured, including Li with an X-ray photon energy of $54 \mathrm{eV}$ [3].

2. Accuracy: The accuracy of standards-based analysis following the Castaing k-ratio protocol, where $\mathrm{k}$

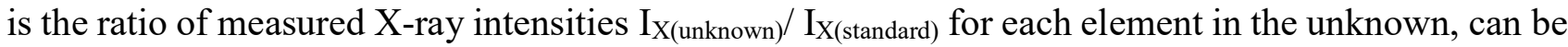
tested by analyzing homogeneous multi-element materials of known composition. In such studies, the accuracy is quantified with the parameter "Relative Deviation from Expected Value (RDEV)", which is defined as

$\mathrm{RDEV}=[($ Measured Value - True Value $) /$ True Value $] \times 100 \%$

The RDEV distribution spans less than $\pm 5 \%$ relative for $95 \%$ of EDS analyses of major (mass concentration $\left.C_{m}>0.1\right)$ and minor $\left(0.01 \leq C_{m} \leq 0.1\right)$ constituents [4]. Accuracy within $\pm 10 \%$ relative extends even to low atomic number elements, at least down to B [5].

3. Precision: The high throughput that is now routine with the silicon drift detector (SDD)-EDS, e.g., an output count rate (OCR) of 100,000 counts per second or more, enables a peak intensity measurement precision of $\sqrt{ } \mathrm{n} / \mathrm{n}=0.1 \%$ or better for major constituents $\left(C_{m}>0.1\right)$ within a practical measurement time of $100 \mathrm{~s}$.

4. Trace constituent measurements: With $100 \mathrm{~s}$ of spectrum accumulation at $100 \mathrm{kHz}$ OCR (yielding 10 million counts integrated from $\sim 50 \mathrm{eV}$ to the incident beam energy, $\mathrm{E}_{0}$ ), the mass concentration limit of detection, $\mathrm{C}_{\mathrm{DL}}$, can reach as low as $\mathrm{C}_{\mathrm{DL}}=0.00025$ (250 parts per million, ppm) in the absence of peak interference [6]. When severe peak interference from a higher concentration constituent occurs, trace levels as low as $\mathrm{C}_{\mathrm{DL}}=0.001(1000 \mathrm{ppm})$ can still be reached. Increasing the dose can further reduce these values by a factor of two or more.

5. Information per unit dose: Comparison of measurements of the intensities for copper $\left(\mathrm{Cu} K-\mathrm{L}_{2,3}\right.$ and $\mathrm{Cu} \mathrm{L}_{3}-\mathrm{M}_{4,5}$ ) and gold ( $\mathrm{Au} \mathrm{L} \mathrm{L}_{3}-\mathrm{M}_{4,5}$ and $\left.\mathrm{Au} \mathrm{M}_{5}-\mathrm{N}_{6,7}\right)$ ) on NIST SRM 482, made simultaneously with EDS and wavelength dispersive spectrometry (WDS) on an electron microprobe, revealed that EDS requires a factor of 10 to 250 lower dose (beam current $x$ measurement time) than WDS to reach the same count precision, depending on the photon energy being measured [7]. For this particular instrument, the cluster of four $10 \mathrm{~mm}^{2}$ SDD detectors was placed at a distance of $70 \mathrm{~mm}$, giving a solid angle $\Omega=0.0082 \mathrm{sr}$. With four $30 \mathrm{~mm}^{2}$ detectors at $30 \mathrm{~mm}$ in a configuration fully optimized for the EDS solid angle, the EDS dose advantage over WDS could be further improved by at least another factor of fifteen.

6. Low beam energy microanalysis $\left(1 \mathrm{keV} \leq \mathrm{E}_{0} \leq 5 \mathrm{keV}\right)$ enables high lateral spatial resolution and shallow sampling depth. Interpretation can be challenging due to the complex structure (e.g., surface layers) encountered in many practical materials. Measurements are subject to limitations on sensitivity, the elements that can be accessed diminish as $\mathrm{E}_{0}$ is reduced, and unfamiliar X-ray families must be used, but useful quantitative results can still be achieved [8]. 
7. Detecting the unexpected constituent despite severe peak interference: One of the greatest challenges to an analytical technique is the discovery of the unexpected, particularly when low intensity peaks that arise from minor and trace $\left(\mathrm{C}_{\mathrm{m}}<0.01\right)$ constituents suffer interference from major constituents. Such hidden constituents can be recognized by following an iterative analysis approach which alternates qualitative analysis (i.e., peak identification of the elements present) with quantitative analysis that includes inspection of the peak fitting "residual spectrum" after each iteration [9]. An example is shown in Figure 1 for NIST glass K873, in which a trace level of $\mathrm{Ce}\left(\mathrm{C}_{\mathrm{m}}=0.0041\right)$ suffers severe peak interference from barium $\left(\mathrm{C}_{\mathrm{m}}=0.219\right)$, a mass concentration ratio of $\mathrm{Ba} / \mathrm{Ce}=53$. The $\mathrm{Ce}$ $\mathrm{L}_{3}-\mathrm{M}_{4,5}$ peak is separated from the $\mathrm{Ba} \mathrm{L}_{2}-\mathrm{M}_{4}$ peak by $12 \mathrm{eV}$. Using NIST DTSA-II, the peak fitting residual spectrum is shown in Figure 1 with and without fitting for Ce [10]. The peaks resulting from the trace level of $\mathrm{Ce}$ are clearly revealed when fitting only for the Ba L-family. The excess intensity for both the $\mathrm{Ce}_{3}-\mathrm{M}_{4,5}$ and the $\mathrm{Ce}_{2}-\mathrm{M}_{4}$ peaks can be seen in the peak fitting residual.

\section{References:}

[1] R. Fitzgerald, R., K. Keil, and K. Heinrich, Science, 525 (1968), p. 159.

[2] J. Goldstein et al, in "Scanning Electron Microscopy and X-ray Microanalysis", $4^{\text {th }}$ ed. (Springer, New York).

[3] L. Xiaobing et al, Micros. Microanal. 19 (S2) (2013), p. 1136.

[4] D. Newbury and N. Ritchie, J. Mats. Sci. 50 (2015), p. 493.

[5] D. Newbury and N. Ritchie, Micros. Microanal. 21 (2015), p. 1327.

[6] D. Newbury and N. Ritchie, Micros. Microanal. 22 (2016), p. 520.

[7] N. Ritchie, D. Newbury, and J. Davis, Micros. Microanal. 18 (2012), p. 892.

[8] D. Newbury and N. Ritchie, Micros. Microanal. 22 (2016), p. 735.

[9] D. Newbury and N. Ritchie, Micros. Microanal. 22 (S2) (2016), p. 396.

[10] N. Ritchie, DTSA-II available free at: www.cstl.nist.gov/div837/837.02/epq/dtsa2/index.html
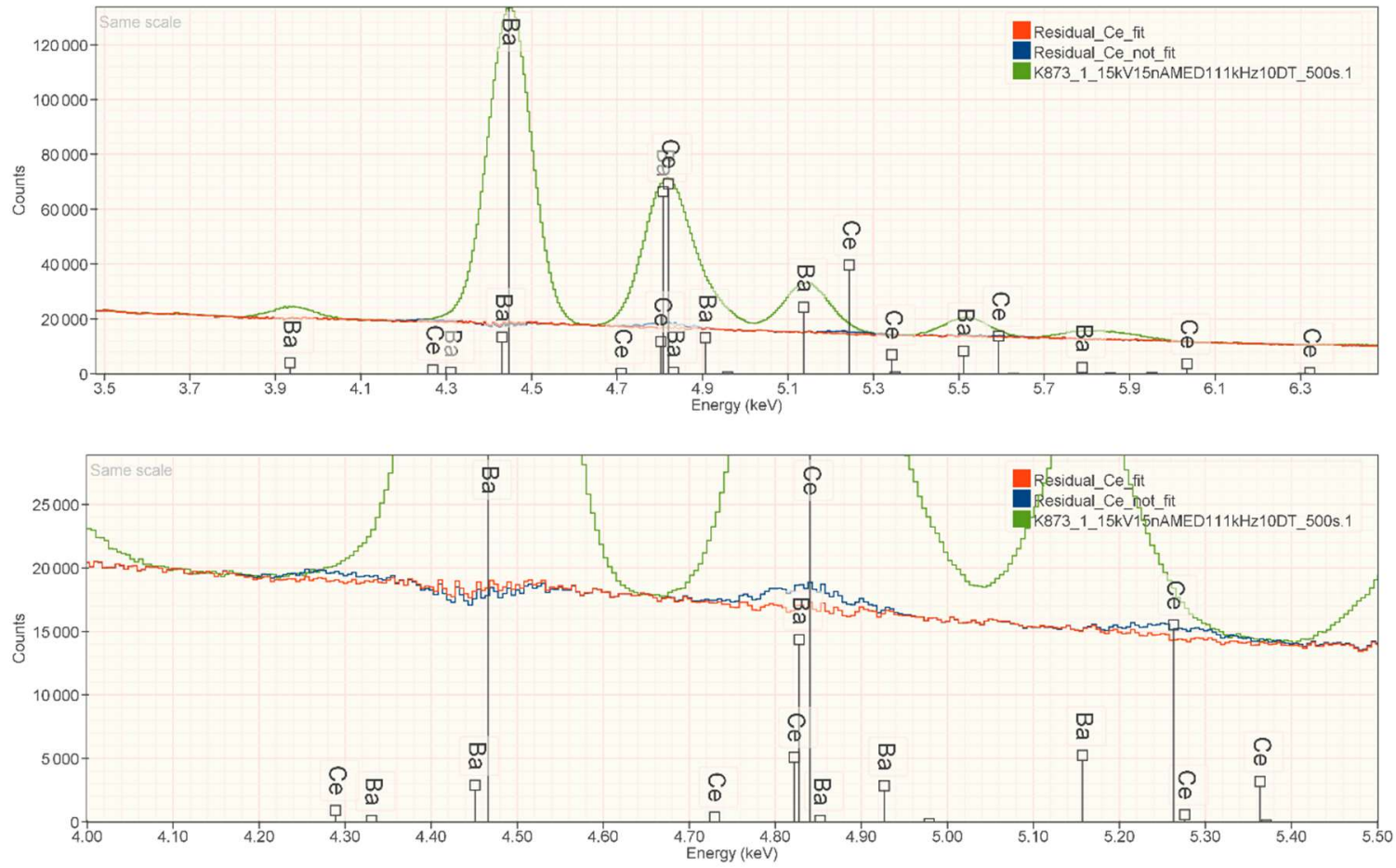

Figure 1. NIST glass K873 ( $\mathrm{E}_{0}=15 \mathrm{keV} ; 55$ million counts) spectrum (green trace) and the peak fitting residual spectrum after fitting for $\mathrm{Ba}$ only (blue trace) and for both $\mathrm{Ba}$ and $\mathrm{Ce}$ (red trace). The $\mathrm{Ba} / \mathrm{Ce}$ concentration ratio is 53, with Ce present at 0.0041 mass concentration (4100 ppm). 